PROCEEDINGS OF THE

AMERICAN MATHEMATICAL SOCIETY

Volume 133, Number 4, Pages 1075-1081

S 0002-9939(04)07791-3

Article electronically published on November 1, 2004

\title{
RESTRICTED WEAK TYPE VERSUS WEAK TYPE
}

\author{
LOUKAS GRAFAKOS AND MIECZYSŁAW MASTYŁO
}

(Communicated by Andreas Seeger)

\begin{abstract}
We prove that translation invariant multilinear operators of restricted weak type $(1,1, \ldots, 1, q)$ must necessarily be of weak type $(1,1, \ldots$, $1, q)$. We give applications.
\end{abstract}

\section{INTRODUCTION AND THE MAIN RESUlT}

Let $X$ be a normed (or quasi-normed) linear space of functions defined on a measure space $(M, \mu)$. A linear (or sublinear) operator $T$ defined on $X$ and taking values in $L^{q, \infty}(N)$ (weak $L^{q}$ of a measure space $\left.(N, \nu)\right), 0<q<\infty$, is said to be of restricted weak type $(X, q)$ if there is a constant $C$ such that for every characteristic function $\chi_{A}$ in $X(A$ is a measurable subset of $M)$ we have

$$
\left\|T\left(\chi_{A}\right)\right\|_{L^{q, \infty}(N)}=: \sup _{\lambda>0}\left[\lambda \nu\left(\left\{x \in N:\left|T\left(\chi_{A}\right)(x)\right|>\lambda\right\}\right)^{\frac{1}{q}}\right] \leq C\left\|\chi_{A}\right\|_{X} .
$$

In the special case where $X=L^{p}(M)$ we say that $T$ is of restricted weak type $(p, q)$. A pair of restricted weak type estimates are powerful enough to often imply strong type estimates on intermediate spaces. Restricted weak type estimates are usually easier to obtain than strong type estimates as the functions involved are bounded and two-valued instead of arbitrary measurable.

The general question we are concerned with is under what conditions on $X$ and $T$ does the restricted weak type $(X, q)$ estimate (1) imply the full weak type estimate

$$
\|T(f)\|_{L^{q, \infty}(N)}=\sup _{\lambda>0}\left[\lambda \nu(\{x \in N:|T(f)(x)|>\lambda\})^{\frac{1}{q}}\right] \leq C^{\prime}\|f\|_{X}
$$

for all functions $f$ in $X$. Here $C^{\prime}$ is a constant that is allowed to depend only on $C, q$ and the space $X$. It is known that a general linear operator $T$ of restricted weak type $(p, q)$ is not necessarily of weak type $(p, q)$. Stein and Weiss [SW] considered the linear operator

$$
S(f)(x)=x^{-1 / q} \int_{0}^{\infty} y^{-1 / p^{\prime}} f(y) d y,
$$

defined for functions on $(0, \infty)$, to indicate that a restricted weak type $(p, q)$ property does not necessarily imply the corresponding weak type $(p, q)$ property. Here

Received by the editors November 6, 2003.

2000 Mathematics Subject Classification. Primary 46B70, 42 B99.

Key words and phrases. Multilinear interpolation, restricted weak type estimates.

The first author was supported by the National Science Foundation under grant DMS 0099881.

The second author was supported by KBN Grant 1 P03A 01326.

(C)2004 American Mathematical Society Reverts to public domain 28 years from publication 
$1<p, q<\infty$ and $p^{\prime}$ is defined by $1 / p+1 / p^{\prime}=1$. A remarkable theorem of Moon [M], however, says that if a convolution operator on $L^{1}\left(\mathbf{R}^{n}\right)$ is of restricted weak type $(1, q)$, then it must necessarily be of weak type $(1, q)$. This theorem is also valid for maximal convolution operators:

Theorem (Moon $[\mathrm{M}]$ ). Let $S_{j}, j=1,2, \ldots$, be linear operators on $L^{1}\left(\mathbf{R}^{n}\right)$ each of the form $S_{j}(f)=f * K_{j}$ for some $K_{j}$ in $L^{1}\left(\mathbf{R}^{n}\right)$ and let

$$
S_{*}(f)=\sup _{j \geq 1}\left|S_{j}(f)\right| .
$$

If $S_{*}$ is of restricted weak type $(1, q)$ for some $q>0$, then $S_{*}$ must be of weak type $(1, q)$ with constant independent of the quantities $\left\|K_{j}\right\|_{L^{1}}$.

The hypothesis that each $K_{j}$ is integrable may seem very strong. In most applications, nevertheless, one can work equally well with an integrable truncation of the kernel $K_{j}$ and obtain restricted weak type estimates independent of the truncation. Moon's theorem then yields weak type estimates independent of the truncation and, passing to the limit (using Fatou's lemma for weak spaces), one obtains weak type estimates for the actual operator. Here is an example: Let $I_{\alpha}$ be the usual fractional integral operator on $\mathbf{R}^{n}$ given by convolution with the kernel $|x|^{-n+\alpha}$. We can use the previous theorem to show that $I_{\alpha}$ maps $L^{1}\left(\mathbf{R}^{n}\right)$ to $L^{n /(n-\alpha), \infty}\left(\mathbf{R}^{n}\right)$ when $0<\alpha<n$. (Using duality and interpolation, this fact implies that $I_{\alpha}$ maps $L^{p}\left(\mathbf{R}^{n}\right)$ to $L^{q}\left(\mathbf{R}^{n}\right)$ whenever $1 / p-1 / q=\alpha / n$.) Using Moon's theorem it will suffice to show that the operator given by convolution with the truncated integrable kernel $|x|^{-n+\alpha} \chi_{|x| \leq B}$ is of restricted weak type $(1, n /(n-\alpha))$ (with constant independent of the parameter $B$ ). But this amounts to showing that for some dimensional constant $C_{n}$ and all measurable sets $E, F$ with finite Lebesgue measure (denoted by $|E|$ and $|F|)$ one has

$$
\int_{F} \int_{E}|x-t|^{-n+\alpha} \chi_{|x-t| \leq B} d t d x \leq C_{n}|E||F|^{n / \alpha}
$$

for all $B>0$. Applying Fubini's theorem and noting that $\int_{F}|x-t|^{-n+\alpha} d x \leq$ $C_{n}|F|^{n / \alpha}$ for all $x$ in $\mathbf{R}^{n}$, (3) follows.

In this work we prove a multilinear version of Moon's theorem. Our result will also be limited to multilinear "convolution" operators, which are usually called "translation invariant" in this context. These are multilinear operators of the form

$$
T\left(f_{1}, \ldots, f_{m}\right)(x)=\int_{\left(\mathbf{R}^{n}\right)^{m}} K\left(x, y_{1}, \ldots, y_{m}\right) f_{1}\left(y_{1}\right) \ldots f_{m}\left(y_{m}\right) d y_{1} \ldots d y_{m}
$$

where the kernel $K\left(x, y_{1}, \ldots, y_{m}\right)$ has the form $K_{0}\left(x-y_{1}, \ldots, x-y_{m}\right)$ for some function (or distribution) $K_{0}$ of one less variable. As in Moon's theorem we will work with a supremum of translation invariant operators. Inspired by the linear case we introduce the following terminology: we say that a multilinear (or multisublinear) operator $T$ is of restricted weak type $\left(p_{1}, \ldots, p_{m}, q\right)$ if for all measurable sets $E_{1}, \ldots, E_{m}$ of finite measure we have

$$
\left\|T\left(\chi_{E_{1}}, \ldots, \chi_{E_{m}}\right)\right\|_{L^{q, \infty}} \leq A\left|E_{1}\right|^{\frac{1}{p_{1}}} \ldots\left|E_{m}\right|^{\frac{1}{p_{m}}}
$$

for some positive constant $A$. The following is our main result. 
Theorem 1.1. For $j=1,2, \ldots$, let $T_{j}$ be an $m$-linear translation invariant operator on $L^{1}\left(\mathbf{R}^{n}\right) \times \cdots \times L^{1}\left(\mathbf{R}^{n}\right)$ with kernel an integrable bounded function $K_{j}$ on $\left(\mathbf{R}^{n}\right)^{m}$ and let

$$
T_{*}\left(f_{1}, \ldots, f_{m}\right)=\sup _{j \geq 1}\left|T_{j}\left(f_{1}, \ldots, f_{m}\right)\right| .
$$

Let $0<q<\infty$. If $T_{*}$ is of restricted weak type $(1, \ldots, 1, q)$, then $T_{*}$ must be of weak type $(1, \ldots, 1, q)$ with constant independent of the quantities $\left\|K_{j}\right\|_{L^{1}},\left\|K_{j}\right\|_{L^{\infty}}$.

We prove this theorem in the next section and we discuss a few applications in the last section.

\section{THE PROOF OF THE MAIN RESULT}

Let us denote by $C_{c}\left(\mathbf{R}^{n}\right)$ the set of all continuous functions on $\mathbf{R}^{n}$ with compact support and by $S\left(\mathbf{R}^{n}\right)$ the set of all simple functions each of which is a finite linear combination of characteristic functions of compact connected sets.

Let us fix an $m$-tuple $\left(f_{1}, \ldots, f_{m}\right)$ of nonnegative functions in $S\left(\mathbf{R}^{n}\right)^{m}$. Once the required estimate is proved for such functions, it is easily extended for all complexvalued functions using multilinearity (with an extra factor of $4^{m}$ in the constant).

Since $S\left(\left(\mathbf{R}^{n}\right)^{m}\right)$ is dense in $L^{1}\left(\left(\mathbf{R}^{n}\right)^{m}\right)$, given an $\varepsilon>0$, there exist functions $H_{j}$ in $S\left(\left(\mathbf{R}^{n}\right)^{m}\right)$ such that

$$
\left\|H_{j}-K_{j}\right\|_{L^{1}} \leq \frac{\varepsilon}{2 \max \left(1,\left\|f_{1}\right\|_{L^{\infty}} \ldots\left\|f_{m}\right\|_{L^{\infty}}\right)} .
$$

Setting

$$
\widetilde{T}_{j}\left(f_{1}, \ldots, f_{m}\right)=\int_{\mathbf{R}^{m n}} H_{j}\left(x-y_{1}, \ldots, x-y_{m}\right) f_{1}\left(y_{1}\right) \ldots f_{m}\left(y_{m}\right) d y_{1} \ldots d y_{m},
$$

we have

$$
\begin{aligned}
& \left|T_{j}\left(f_{1}, \ldots, f_{m}\right)-\widetilde{T}_{j}\left(f_{1}, \ldots, f_{m}\right)\right| \\
= & \mid \int_{\mathbf{R}^{m n}}\left[H_{j}\left(x-y_{1}, \ldots, x-y_{m}\right)\right. \\
& \left.-K_{j}\left(x-y_{1}, \ldots, x-y_{m}\right)\right] f_{1}\left(y_{1}\right) \ldots f_{m}\left(y_{m}\right) d y_{1} \ldots d y_{m} \mid \\
\leq & \left\|f_{1}\right\|_{L^{\infty}} \ldots\left\|f_{m}\right\|_{L^{\infty}}\left\|K_{j}-H_{j}\right\|_{L^{1}}<\frac{\varepsilon}{2} .
\end{aligned}
$$

Let us fix a positive integer $J$. For any fixed $\lambda>0$ and all positive integers $j$, $1 \leq j \leq J$, the continuity of $H_{j}$ implies the existence of a $\delta>0$ such that for any connected set $\mathbf{I}$ in $\mathbf{R}^{m n}$ with

$$
\operatorname{diam}(\mathbf{I})=\sup \{|\mathbf{x}-\mathbf{y}|: \mathbf{x}, \mathbf{y} \in \mathbf{I}\}<\delta
$$

we have

$$
\left|H_{j}(\mathbf{y})-H_{j}(\mathbf{z})\right|<\frac{\lambda}{2\left\|f_{1}\right\|_{L^{1}} \ldots\left\|f_{m}\right\|_{L^{1}}}
$$

whenever $\mathbf{y}, \mathbf{z} \in \mathbf{I}$ for all $1 \leq j \leq J$. (We will use boldface letters for points in $\mathbf{R}^{m n}$.)

For each $i \in\{1, \ldots, m\}$ we write $\mathbf{R}^{n}$ as a countable disjoint union of connected sets $I_{i, k_{i}}$ such that

$$
\operatorname{diam}\left(I_{1, k_{1}} \times \cdots \times I_{m, k_{m}}\right)<\delta .
$$


Since each $f_{i}$ is a simple function, we may write

$$
f_{i}(x)=\sum_{k_{i}} \alpha_{i, k_{i}} \chi_{I_{i, k_{i}}} .
$$

There are only finitely many nonzero $\alpha_{i, k_{i}}$ and we set $\alpha_{i}=\max _{k_{i}}\left(\alpha_{i, k_{i}}\right)$ for all $i \in\{1,2, \ldots, n\}$. We clearly have $\alpha_{i}=\left\|f_{i}\right\|_{L^{\infty}}$.

For each $1 \leq i \leq m$ and $k_{i}$ we pick a subset $F_{i, k_{i}}$ of $I_{i, k_{i}}$ such that

$$
\alpha_{i}\left|F_{i, k_{i}}\right|=\alpha_{i, k_{i}}\left|I_{i, k_{i}}\right| .
$$

We now set $E_{i}=\bigcup_{k_{i}} F_{i, k_{i}}, \mathbf{k}=\left(k_{1}, \ldots, k_{m}\right), \mathbf{F}_{\mathbf{k}}=F_{1, k_{1}} \times \cdots \times F_{m, k_{m}}, \mathbf{I}_{\mathbf{k}}=$ $I_{1, k_{1}} \times \cdots \times I_{m, k_{m}}$, and using (7) we note that for all $i$ in $\{1, \ldots, m\}$ the following is valid:

$$
\alpha_{i}\left|E_{i}\right|=\sum_{k_{i}} \alpha_{i}\left|F_{i, k_{i}}\right|=\sum_{k_{i}} \alpha_{i, k_{i}}\left|I_{i, k_{i}}\right|=\left\|f_{i}\right\|_{L^{1}} .
$$

Let $x \in \mathbf{R}^{n}$. Setting $\mathbf{x}^{0}=(x, \ldots, x)$, for each $j \in\{1,2, \ldots, J\}$ we have

$$
\begin{aligned}
& \left|\widetilde{T}_{j}\left(f_{1}, \ldots, f_{m}\right)(x)-\widetilde{T}_{j}\left(\alpha_{1} \chi_{E_{1}}, \ldots, \alpha_{m} \chi_{E_{m}}\right)(x)\right| \\
\leq & \sum_{k_{1}} \cdots \sum_{k_{m}} \mid \widetilde{T}_{j}\left(\alpha_{1, k_{1}} \chi_{I_{1, k_{1}}}, \ldots, \alpha_{m, k_{m}} \chi_{I_{m, k_{m}}}\right)(x) \\
& -\widetilde{T}_{j}\left(\alpha_{1} \chi_{F_{1, k_{1}}}, \ldots, \alpha_{m} \chi_{F_{m, k_{m}}}\right)(x) \mid \\
= & \sum_{k_{1}} \cdots \sum_{k_{m}}\left|\alpha_{1, k_{1}} \ldots \alpha_{m, k_{m}} \int_{\mathbf{I}_{\mathbf{k}}} H_{j}\left(\mathbf{x}^{0}-\mathbf{y}\right) d \mathbf{y}-\alpha_{1} \ldots \alpha_{m} \int_{\mathbf{F}_{\mathbf{k}}} H_{j}\left(\mathbf{x}^{0}-\mathbf{y}\right) d \mathbf{y}\right| \\
= & \sum_{k_{1}} \cdots \sum_{k_{m}}\left|\alpha_{1, k_{1}} \ldots \alpha_{m, k_{m}}\right| \mathbf{I}_{\mathbf{k}}\left|H_{j}\left(\mathbf{x}^{0}-\mathbf{y}_{\mathbf{k}}\right)-\alpha_{1} \ldots \alpha_{m}\right| \mathbf{F}_{\mathbf{k}}\left|H_{j}\left(\mathbf{x}^{0}-\mathbf{y}_{\mathbf{k}}^{\prime}\right)\right| \\
= & \sum_{k_{1}} \cdots \sum_{k_{m}}\left|\alpha_{1} \ldots \alpha_{m}\right| \mathbf{F}_{\mathbf{k}}\left|H_{j}\left(\mathbf{x}^{0}-\mathbf{y}_{\mathbf{k}}\right)-\alpha_{1} \ldots \alpha_{m}\right| \mathbf{F}_{\mathbf{k}}\left|H_{j}\left(\mathbf{x}^{0}-\mathbf{y}_{\mathbf{k}}^{\prime}\right)\right|
\end{aligned}
$$

for some $y_{\mathbf{k}}$ in $\mathbf{I}_{\mathbf{k}}$ and $y_{\mathbf{k}}^{\prime}$ in $\mathbf{F}_{\mathbf{k}}$, as a consequence of the mean value theorem. In the last identity we used (7). As the diameter of the set $\mathbf{x}^{0}-\mathbf{I}_{\mathbf{k}}$ is at most $\delta$, using (6) we obtain that the last displayed expression above is at most

$$
\sum_{k_{1}} \cdots \sum_{k_{m}} \alpha_{1} \ldots \alpha_{m}\left|\mathbf{F}_{\mathbf{k}}\right| \frac{\lambda}{2\left\|f_{1}\right\|_{L^{1}} \ldots\left\|f_{m}\right\|_{L^{1}}}=\frac{\lambda}{2} .
$$

Combining the results of the previous calculations we obtain

$$
\begin{aligned}
\left|T_{j}\left(f_{1}, \ldots, f_{m}\right)\right| & \leq\left|\widetilde{T}_{j}\left(f_{1}, \ldots, f_{m}\right)\right|+\frac{\varepsilon}{2} \\
& \leq\left|\widetilde{T}_{j}\left(\alpha_{1} \chi_{E_{1}}, \ldots, \alpha_{m} \chi_{E_{m}}\right)\right|+\frac{\lambda}{2}+\frac{\varepsilon}{2} \\
& \leq\left|T_{j}\left(\alpha_{1} \chi_{E_{1}}, \ldots, \alpha_{m} \chi_{E_{m}}\right)\right|+\frac{\varepsilon}{2}+\frac{\lambda}{2}+\frac{\varepsilon}{2} \\
& \leq \alpha_{1} \ldots \alpha_{m} T_{*}\left(\chi_{E_{1}}, \ldots, \chi_{E_{m}}\right)+\frac{\lambda}{2}+\varepsilon
\end{aligned}
$$

and from this we deduce that

$$
\left|\left\{\sup _{1 \leq j \leq J}\left|T_{j}\left(f_{1}, \ldots, f_{m}\right)\right|>\lambda+\varepsilon\right\}\right| \leq\left|\left\{\left|T_{*}\left(\chi_{E_{1}}, \ldots, \chi_{E_{m}}\right)\right|>\frac{\lambda}{2 \alpha_{1} \ldots \alpha_{m}}\right\}\right| .
$$


Using our assumption that the operator $T_{*}$ is of restricted weak type $(1, \ldots, 1, q)$ we conclude that the last expression in (8) is at most

$$
\left(A\left|E_{1}\right| \ldots\left|E_{m}\right| 2 \alpha_{1} \ldots \alpha_{m} \lambda^{-1}\right)^{q}=\left(2 A\left\|f_{1}\right\|_{L^{1}} \ldots\left\|f_{m}\right\|_{L^{1} \lambda^{-1}}\right)^{q} .
$$

Letting $J \rightarrow \infty$ and $\varepsilon \rightarrow 0$ we obtain that $T_{*}$ satisfies the required weak type estimate for all functions $f_{1}, \ldots, f_{m}$ in $S\left(\mathbf{R}^{n}\right)$.

It remains to consider general functions in $L^{1}\left(\mathbf{R}^{n}\right)$. As the operator $T_{*}$ is not linear, this extension is not automatic. For the rest of this proof, we fix functions $f_{1}, \ldots, f_{m}$ in $L^{1}\left(\mathbf{R}^{n}\right)$.

Using the multilinearity of the $T_{j}$ we can easily show that there is a constant $C$ (depending on $m$ variables) such that for all integrable functions $f_{j}, g_{j}$ satisfying $\left\|f_{j}-g_{j}\right\|_{L^{1}}<1$ we have

$$
\begin{aligned}
& \left\|T_{j}\left(f_{1}, \ldots, f_{m}\right)-T_{j}\left(g_{1}, \ldots, g_{m}\right)\right\|_{L^{1}} \\
& \quad \leq\left\|K_{j}\right\|_{L^{\infty}} C\left(\left\|f_{1}\right\|_{L^{1}} \ldots,\left\|f_{m}\right\|_{L^{1}}\right)\left[\sum_{i=1}^{m}\left\|f_{i}-g_{i}\right\|_{L^{1}}\right] .
\end{aligned}
$$

Let $J$ be a fixed positive integer. For any given $0<\varepsilon<1$ we can find functions $g_{j}$ (depending on $\varepsilon, J)$ in $S\left(\mathbf{R}^{n}\right)$ such that

$$
\left\|f_{j}-g_{j}\right\|_{L^{1}}<\varepsilon^{2}\left[\max \left(1, C\left(\left\|f_{1}\right\|_{L^{1}}, \ldots,\left\|f_{m}\right\|_{L^{1}}\right) \max \left(\left\|K_{1}\right\|_{L^{\infty}}, \ldots,\left\|K_{J}\right\|_{L^{\infty}}\right)\right)\right]^{-1} \text {. }
$$

Then for each $j \in\{1, \ldots, J\}$ we have

$$
\left\|T_{j}\left(f_{1}, \ldots, f_{m}\right)-T_{j}\left(g_{1}, \ldots, g_{m}\right)\right\|_{L^{1}}<\varepsilon^{2},
$$

which, via Chebychev's inequality, implies that the set

$$
B_{j}=\left\{\left|T_{j}\left(f_{1}, \ldots, f_{m}\right)-T_{j}\left(g_{1}, \ldots, g_{m}\right)\right|>\varepsilon\right\}
$$

has measure at most $\varepsilon$. Letting $B(J)=\bigcup_{j=1}^{J} B_{j}$, for all $x \notin B(J)$ we have

$$
\sup _{1 \leq j \leq J}\left|T_{j}\left(f_{1}, \ldots, f_{m}\right)\right| \leq \sup _{1 \leq j \leq J}\left|T_{j}\left(g_{1}, \ldots, g_{m}\right)\right|+\varepsilon \leq T_{*}\left(g_{1}, \ldots, g_{m}\right)+\varepsilon .
$$

It follows that

$$
\begin{aligned}
& \left|\left\{\sup _{1 \leq j \leq J}\left|T_{j}\left(f_{1}, \ldots, f_{m}\right)\right|>\lambda+\varepsilon\right\}\right| \leq\left|\left\{T_{*}\left(g_{1}, \ldots, g_{m}\right)>\lambda\right\}\right|+|B(J)| \\
& \quad \leq\left(A \lambda^{-1}\left\|g_{1}\right\|_{L^{1}} \ldots\left\|g_{m}\right\|_{L^{1}}\right)^{q}+\sum_{j=1}^{J}\left|B_{j}\right| \\
& \quad \leq\left(A \lambda^{-1}\left(\left\|f_{1}\right\|_{L^{1}}+\varepsilon^{2}\right) \ldots\left(\left\|f_{m}\right\|_{L^{1}}+\varepsilon^{2}\right)\right)^{q}+J \varepsilon .
\end{aligned}
$$

Letting first $\varepsilon \rightarrow 0$ and then $J \rightarrow \infty$ we conclude that

$$
\left|\left\{T_{*}\left(f_{1}, \ldots, f_{m}\right)>\lambda\right\}\right| \leq\left(A \lambda^{-1}\left\|f_{1}\right\|_{L^{1}} \ldots\left\|f_{m}\right\|_{L^{1}}\right)^{q} .
$$

Remark 2.1. Suppose that $K_{j}=0$ for all $j \geq 2$. Then the assumption that $K_{1}$ is in $L^{\infty}\left(\left(\mathbf{R}^{n}\right)^{m}\right)$ can be dropped. In this case, the following is valid: If $T_{1}$ is of restricted weak type $(1, \ldots, 1, q)$, then $T_{1}$ must be of weak type $(1, \ldots, 1, q)$ with constant independent of the quantity $\left\|K_{1}\right\|_{L^{1}}$. Indeed, in this case, the passage from $S\left(\mathbf{R}^{n}\right)$ to $L^{1}\left(\mathbf{R}^{n}\right)$ follows by a simple density argument in view of the (multi)linearity of $T_{1}$. 


\section{Applications}

Let $H$ be the Hilbert transform and $F$ be a measurable subset of $\mathbf{R}$ of finite measure. It is shown in $\mathrm{SW}$ that

$$
\left|\left\{x \in \mathbf{R}:\left|H\left(\chi_{F}\right)(x)\right|>\lambda\right\}\right|=\frac{4|F|}{e^{\pi \lambda}-e^{-\pi \lambda}}
$$

for all $\lambda>0$. Observing that this function is at most a multiple of $\lambda^{-1}$ and using Moon's theorem $[\mathrm{M}$, we conclude that $H$ is of weak type $(1,1)$. Although a precise identity is not known in the $m$-linear case, an estimate that captures the whole essence of (9) for multilinear Calderón-Zygmund operators is contained in a forthcoming publication by Bilyk and the first author [BG]: Let $T_{0}$ be a translation invariant $m$-linear Calderón-Zygmund operator on $\mathbf{R}^{n} \times \cdots \times \mathbf{R}^{n}$ (see GT for the pertinent definitions and for a short account of the general theory). Then there is a constant $C$ (depending only on $m$ and $n$ ) such that for all sets $F_{1}, \ldots, F_{m}$ of finite measure we have

$$
\left|\left\{\left|T_{0}\left(\chi_{F_{1}}, \ldots, \chi_{F_{m}}\right)\right|>\lambda\right\}\right| \leq C\left(\left|F_{1}\right| \ldots\left|F_{m}\right|\right)^{-1 / m} \phi(\lambda),
$$

where

$$
\phi(\lambda)= \begin{cases}\lambda^{-1 / m} & \text { when } \lambda<1, \\ e^{-c \lambda} & \text { when } \lambda \geq 1,\end{cases}
$$

and $c$ is another constant that depends only on $m$ and $n$. In particular, the previous estimate implies that $T_{0}$ is of restricted weak type $(1, \ldots, 1,1 / m)$. Applying Theorem 1.1 we deduce that $T_{0}$ is of weak type $(1, \ldots, 1,1 / \mathrm{m})$; this fact is already contained in GT], and we discuss here an alternative approach.] There is a small technical issue concerning the kernel of $T_{0}$ that needs to be addressed here; this argument provides a typical illustration of the way one handles problems of similar nature in the application of Theorem 1.1 The kernel of an $m$-linear CalderónZygmund operator is a distribution $K_{0}$ of $m n$ real variables that coincides with a function satisfying

$$
\left|K_{0}\left(x_{1}, \ldots, x_{m}\right)\right| \leq A\left(\sum_{i, j=1}^{m}\left|x_{i}-x_{j}\right|\right)^{-m n}
$$

for all $\left(x_{1}, \ldots, x_{m}\right)$ away from the diagonal in $\mathbf{R}^{m n}$ and also satisfying an analogous estimate for its gradient. As Theorem 1.1 requires $K_{0}$ to be integrable, we will have to consider the integrable truncations

$$
K_{0}^{\varepsilon}\left(x_{1}, \ldots, x_{m}\right)=K_{0}\left(x_{1}, \ldots, x_{m}\right) \chi_{\varepsilon^{2} \leq\left|x_{1}\right|^{2}+\cdots+\left|x_{m}\right|^{2} \leq \varepsilon^{-2}}
$$

of $K_{0}$ defined for $\varepsilon<1$. Estimate (10) also holds for the operator $T_{0}^{\varepsilon}$ with kernel $K_{0}^{\varepsilon}$ with constant independent of $\varepsilon$; applying Theorem 1.1 we deduce that the $T_{0}^{\varepsilon}$ 's are of weak type $(1, \ldots, 1,1 / m)$ with constants independent of $\varepsilon$. Passing to the limit and using Fatou's theorem for weak type spaces we obtain that $T_{0}$ is of weak type $(1, \ldots, 1,1 / m)$.

\footnotetext{
${ }^{1}$ Estimate (10) can be derived without the weak type $(1, \ldots, 1,1 / m)$ property of $T_{0}$. For simplicity, in $[\overrightarrow{B G}]$ it was proved using this property, but we point out that this was not necessary.
} 
For a second application, we consider the mixed-homogeneity fractional integral operator

$$
\begin{aligned}
& I_{\gamma_{1}, \ldots, \gamma_{m}, \gamma}\left(f_{1}, \ldots, f_{m}\right)(x) \\
& \quad=\int_{\mathbf{R}^{n}} \ldots \int_{\mathbf{R}^{n}} \prod_{i=1}^{m} f_{i}\left(x-t_{i}\right)\left(\left|t_{1}\right|^{\gamma_{1}}+\cdots+\left|t_{m}\right|^{\gamma_{m}}\right)^{-\gamma} d t_{1} \ldots d t_{m} .
\end{aligned}
$$

We have the following:

Proposition 3.1. Assuming $0<\min \left(\gamma_{1}, \ldots, \gamma_{m}\right)<n / \gamma$, the operator $I_{\gamma_{1}, \ldots, \gamma_{m}, \gamma}$ maps $L^{1}\left(\mathbf{R}^{n}\right) \times \cdots \times L^{1}\left(\mathbf{R}^{n}\right)$ into $L^{\frac{n}{\gamma \mu}, \infty}\left(\mathbf{R}^{n}\right)$ where $\mu=\max \left(\gamma_{1}, \ldots, \gamma_{m}\right)$.

To be able to apply Theorem 1.1 we will insert the truncation $\left|t_{1}\right|+\cdots+\left|t_{m}\right| \leq N$ in the kernel of the operator and will obtain estimates independent of $N$. Using Theorem 1.1 and a simple characterization of weak $L^{p}$, it will suffice to show that for all measurable sets $F, E_{1}, \ldots, E_{m}$ of finite measure one has

$\int_{F} \int_{E_{1}} \ldots \int_{E_{m}}\left(\left|x-t_{1}\right|^{\gamma_{1}}+\cdots+\left|x-t_{m}\right|^{\gamma_{m}}\right)^{-\gamma} d t_{1} \ldots d t_{m} d x \leq C\left|E_{1}\right| \ldots\left|E_{m}\right||F|^{1-\frac{n}{\gamma \mu}}$

for some constant $C>0$, where the extra assumption $\left|x-t_{1}\right|+\cdots+\left|x-t_{m}\right| \leq N$ was conveniently dropped at this point. We apply Fubini's theorem and a simple estimate to bound the left-hand side of (11) by

$$
\int_{E_{1}} \ldots \int_{E_{m}} \int_{F}\left|x-t_{j_{0}}\right|^{-\mu \gamma} d x d t_{1} \ldots d t_{m}
$$

where $\gamma_{j_{0}}=\mu=\max \left(\gamma_{1}, \ldots, \gamma_{m}\right)$. As $\int_{F}\left|x-t_{j_{0}}\right|^{-\mu \gamma} d x$ becomes largest when $F$ is a ball of radius $|F|^{1 / n}$ centered at $t_{j_{0}}$, this integral is easily seen to be at most a constant multiple of $|F|^{1-\frac{n}{\gamma \mu}}$, and this clearly implies (11).

\section{REFERENCES}

[BG] D. Bilyk and L. Grafakos, Interplay between distributional estimates and boundedness of operators, submitted.

[GT] L. Grafakos and R. Torres, Multilinear Calderón-Zygmund theory, Adv. in Math. 165 (2002), 124-164. MR 1880324 (2002j:42029)

[M] K. H. Moon, On restricted weak type (1,1), Proc. Amer. Math. Soc. 42 (1974), 148-152. MF 0341196 (49:5946)

[SW] E. M. Stein and G. Weiss, An extension of a theorem of Marcinkiewicz and some of its applications, J. Math. Mech. 8 (1959), 263-284. MR0107163(21:5888)

Department of Mathematics, University of Missouri, Columbia, Missouri 65211

E-mail address: loukas@math.missouri.edu

Faculty of Mathematics and Computer Science, A. Mickiewicz University - and Institute of Mathematics, Polish Academy of Science (Poznań Branch), Umultowska 87, 61-614 Poznań, Poland

E-mail address: mastylo@amu.edu.pl 